\title{
Polynomial Minimum Root Separation
}

\author{
By Siegfried M. Rump
}

\begin{abstract}
The minimum root separation of an arbitrary polynomial $P$ is defined as the minimum of the distances between distinct (real or complex) roots of $P$. Some asymptotically good lower bounds for the root separation of $P$ are given, where $P$ may have multiple zeros. There are applications in the analysis of complexity of algorithms and in the theory of algebraic and transcendental numbers.
\end{abstract}

1. Introduction. Let $P(x)$ be a polynomial with arbitrary (real or complex) coefficients $a_{i}$ of degree $n>0$ with zeros $\lambda_{i}$, so that

$$
P(x)=\sum_{i=0}^{n} a_{i} \cdot x^{i}=a_{n} \cdot \prod_{i=1}^{n}\left(x-\lambda_{i}\right), \quad a_{n} \neq 0 .
$$

We define $\operatorname{sep}(P)$, the minimum root separation of $P$, by (cf. [CH74])

$$
\operatorname{sep}(P)=\min _{\lambda_{i} \neq \lambda_{j}}\left|\lambda_{i}-\lambda_{j}\right|
$$

and the minimum real root separation of $P$ by

$$
\operatorname{rsep}(P)=\min \left\{\left|\lambda_{i}-\lambda_{j}\right| \text { for real } \lambda_{i} \neq \lambda_{j}\right\}
$$

In case of the nonexistence of two distinct (real) roots we set $\operatorname{sep}(P)=\infty(\operatorname{rsep}(P)=$ $\infty)$.

In analyzing the computing time of an algorithm which isolates the real zeros of an arbitrary polynomial $P$ with real algebraic coefficients (see [Ru76]) the problem of a lower bound for $\operatorname{rsep}(P)$ arose. In detail, it was unsatisfactory that the known lower bounds (see [Ca47], [Gu61], [Ma64], [Gu67] and [CH74]) have a " $-n^{2}$ in the exponent", so $\log \left\{\operatorname{sep}(P)^{-1}\right\}=O\left(n^{2}\right)$ providing that $P$ is not assumed to be squarefree, i.e. may have multiple zeros. Furthermore, the discriminant $D(P)$ (see [vW66]) is involved except in the paper of Güting [Gu67], so that the bounds hold only for polynomials without multiple zeros. The main tool of this paper is to derive asymptotically better bounds without using $D(P)$ to obtain general estimates, including the advantage not to have to restrict attention to polynomials without multiple zeros.

There are applications of the following lemmas and theorems also in the theory of algebraic and transcendental numbers (see [Ge59] and [Sn57]), but we shall not speak of this here.

Received February 1, 1977; revised November 14, 1977.

AMS (MOS) subject classifications (1970). Primary $12 \mathrm{D} 10$.

Key words and phrases. Polynomial zeros, inequality, root separation, computing time analysis. 
2. Some Investigations. Throughout the paper the assumptions about the polynomials change so they are stated in every theorem separately. For $P$ as in (1) we define the size of $P$ as

$$
s=s(P)=|P|_{1}=\sum_{i=0}^{n}\left|a_{i}\right|,
$$

and the degree of $P$ as $\operatorname{deg}(P)=n$ for $a_{n} \neq 0$. In estimations for $\operatorname{sep}(P)$ or $\operatorname{rsep}(P)$ one can assume $n \geqslant 2$. We define

$$
|P|_{2}=\left\{\sum_{i=0}^{n}\left|a_{i}\right|^{2}\right\}^{1 / 2} \text { and }|P|_{\infty}=\max _{0 \leqslant i \leqslant n}\left|a_{i}\right| .
$$

For multivariate polynomials $A \in C\left[x_{1}, \ldots, x_{r}\right], r \geqslant 2$, we can write $A=$ $\sum_{i=0}^{n} B_{i} \cdot x_{r}^{i}$ with $B_{i} \in C\left[x_{1}, \ldots, x_{r-1}\right]$ and define recursively

$$
|A|_{1}=\sum_{i=0}^{n}\left|B_{i}\right|_{1} \text {. }
$$

It is well known that for any polynomial $P$ one can construct a polynomial $P^{*}$ having the roots of $P$ as simple zeros, namely

$$
P^{*}=P / \operatorname{gcd}\left(P, P^{\prime}\right),
$$

where $P^{\prime}$ denotes the first derivative of $P$. If the coefficients of $P$ are rational integers, so are the coefficients of $P *$. Therefore, from every lower bound of $\operatorname{sep}(P)$ or $\operatorname{rsep}(P)$, assuming $P$ to be squarefree and using $D(P)$, one can obtain another for arbitrary $P$ (perhaps having multiple zeros) by replacing $s$ by $2^{k} \cdot s$, where $k=\operatorname{deg}\left(P^{*}\right)$, because $\left|P^{*}\right|_{1} \leqslant 2^{k} \cdot|P|_{1}$ (cf. [Mi74, Theorem 2]). However, the known lower bounds contain a factor like $s^{-n}$, so after applying the above observation we have a $2-n^{2}$ in the worst case.

Note that some of our main results can be sharpened in several ways, e.g. by replacing $s=|P|_{1}$ by $|P|_{2}$ or even $|P|_{\infty}$ or by taking Gaussian integers instead of rational integers. However, no effort was made to do this because only bounds depending on $s$ were needed in the author's special purpose.

We start with a very useful but nevertheless relatively unknown inequality (see [La05] and [Ma60]):

Lemma 1. Let $P$ be an arbitrary complex polynomial of size $s$ with leading coefficient $a_{n}$. Then

$$
\left|a_{n}\right| \cdot \prod_{i=1}^{n} \max \left(1,\left|\lambda_{i}\right|\right) \leqslant s,
$$

where $\lambda_{i}$ are the roots of $P$.

From this lemma one can derive a first root separation bound in a very simple manner.

THEOREM 1. Let $P$ be an arbitrary complex polynomial of size $s$ and degree $n$. With $D=D(P)$ denoting the discriminant of $P$ one has

$$
\operatorname{sep}(P)>\min \left(1,\left|a_{n}\right|\right)^{n(\ln n+1)} \cdot|D| \cdot\left\{(2 n)^{n-1} \cdot s^{n(\ln n+3)}\right\}^{-1} .
$$

If $P$ has integral coefficients, the factor $\min \left(1,\left|a_{n}\right|\right)^{n(\ln n+1)}$ can be omitted. 
Proof. We can choose the notation so that $\left|\lambda_{1}\right| \geqslant\left|\lambda_{2}\right| \geqslant \cdots \geqslant\left|\lambda_{n}\right|$. Therefore, by Lemma 1 we obtain, with $d=s /\left|a_{n}\right|$,

$$
\left|\lambda_{1}\right| \leqslant d, \quad\left|\lambda_{1} \cdot \lambda_{2}\right| \leqslant d \Rightarrow\left|\lambda_{2}\right| \leqslant d^{1 / 2},
$$

because otherwise

$$
\left|\lambda_{1}\right| \geqslant\left|\lambda_{2}\right|>d^{1 / 2} \Rightarrow\left|\lambda_{1} \cdot \lambda_{2}\right| \geqslant\left|\lambda_{2}\right|^{2}>d .
$$

By induction we see that

$$
\left|\lambda_{i}\right| \leqslant d^{1 / i}, \quad 1 \leqslant i \leqslant n .
$$

It is known [vW66, Section 35], that

$$
|D(P)|=\left|a_{n}^{n-2} \cdot \prod_{i=1}^{n} P^{\prime}\left(\lambda_{i}\right)\right|=\left|a_{n}^{2 n-2} \cdot \prod_{i \neq j}\left(\lambda_{i}-\lambda_{j}\right)\right| .
$$

Suppose now

$$
\operatorname{sep}(P)=\left|\lambda_{k}-\lambda_{k^{\prime}}\right|, \quad \text { where } 1 \leqslant k, k^{\prime} \leqslant n
$$

Then

$$
|D(P)|=\left|a_{n}\right|^{n-1} \cdot\left\{\prod_{i=1 ; i \neq k}^{n} P^{\prime}\left(\lambda_{i}\right) \mid\right\}\left\{\prod_{i=1 ; i \neq k, k^{\prime}}^{n}\left|\lambda_{k}-\lambda_{i}\right|\right\} \cdot \operatorname{sep}(P) .
$$

Finally, from $P^{\prime}(x)=\sum_{j=1}^{n} j a_{j} \cdot x^{j-1}$ and (2) we get

$$
\left|P^{\prime}\left(\lambda_{i}\right)\right| \leqslant \sum_{j=1}^{n}\left|j \cdot a_{j} \cdot \lambda_{i}^{j-1}\right| \leqslant n \cdot s \cdot d^{(n-1) / i} .
$$

Combining (2), (3) and (4) with $\left|\lambda_{k}-\lambda_{i}\right| \leqslant\left|\lambda_{k}\right|+\left|\lambda_{i}\right| \leqslant 2 d$ yields

$$
\operatorname{sep}(P)>|D| \cdot\left\{\left|a_{n}\right|^{n-1} \cdot n^{n-1} \cdot s^{n-1} \cdot d^{\mu} \cdot(2 d)^{n-2}\right\}^{-1},
$$

where $\mu \leqslant \sum_{i=1}^{n}(n-1) / i<(n-1) \cdot\{\ln n+\gamma+1 /(2 n)\}<n \cdot\{\ln n+1\}, \gamma=$ 0.577 ... (Euler's constant) from [Kn69, 1.2.7, p. 74]. Putting this in (5), we get the estimation of Theorem 1 .

The presumably very first lower bound of $\operatorname{sep}(P)$ in [Ca47] is derived in a similar way, but without using the very sharp Lemma 1, and gives, therefore, a weaker result. One of the last and best lower bounds of $\operatorname{sep}(P)$ can be found in [Gu67, Theorem 7]. Güting proved this inequality in the same way by splitting the product of the $P^{\prime}\left(\lambda_{i}\right)$ in (3), in another product again using Lemma 1.

We, instead, start with a lower bound of $|P(\beta)|$, where $\beta$ is a given algebraic number and $P(\beta) \neq 0$.

3. Lower Bounds for $\operatorname{rse}(P)$. To obtain asymptotically better results we shall see that for given polynomials $P, Q$ with $P(\beta) \neq 0=Q(\beta)$ a lower bound for the absolute value of $P(\beta)$ plays an important rôle. Indeed $|P(\beta)|$ cannot be arbitrarily small, as shown in [Sn57], [Gu61] and [Gu67]. These estimates either use $\operatorname{Res}(P, Q)$ 
or contain a factor $2^{-n^{2}}$, i.e. assume $P$ and $Q$ have no common roots or are asymptotically weak.

In his paper [Lo73] Loos proposed the use of resultants to construct polynomials having predefined zeros. Doing this, one can derive good lower bounds for several purposes. For the problem above we follow Collins and Loos (cf. [CL76, Theorem 5]):

LEMMA 2. Let $P, Q$ be arbitrary integral polynomials (perhaps having common roots) of degrees $0<m=\operatorname{deg}(P), 0<n=\operatorname{deg}(Q)$ and sizes $e=s(P), f=s(Q)$. If for some (real or complex) $\beta$

$$
P(\beta) \neq 0, \text { but } Q(\beta)=0,
$$

then

$$
|P(\beta)|>\left\{(e+1)^{n} \cdot f^{m}+1\right\}^{-1} .
$$

Proof. Let $R(y)$ be the resultant of $Q(x)$ and $y-P(x)$ with respect to $x$. Then

$$
R(y)=b_{n}^{m} \cdot \prod_{i=1}^{n}\left\{y-P\left(\beta_{i}\right)\right\}
$$

where $b_{n}$ denotes the leading coefficient and $\beta_{i}$ are the roots of $Q$. Now $|y-P(x)|_{1} \leqslant$ $e+1$ and a generalization of Hadamard's determinant theorem (cf. [CH74, Theorem 2]) gives

$$
|R|_{\infty} \leqslant(e+1)^{n} \cdot f^{m}
$$

Let

$$
R(y)=\sum_{i=0}^{n} r_{i} \cdot y^{i}=y^{k} \cdot \sum_{i=k}^{n} r_{i} \cdot y^{i-k} \quad \text { with } r_{k} \neq 0 .
$$

Then the roots of

$$
\bar{R}(y)=\sum_{i=k}^{n} r_{i} \cdot y^{n-i+k}
$$

are the inverses of the roots of $R$. It is well known that, for every root $\alpha=P(\lambda)$ of $R$,

$$
|\alpha|<\left|r_{n}\right|^{-1} \cdot|R|_{\infty}+1 \leqslant|R|_{\infty}+1
$$

holds, because $R$ is, as $P$ and $Q$, an integral polynomial. Hence, for the roots of $\bar{R}$ we have, with (7), (8), (9) and $|R|_{\infty}=|\bar{R}|_{\infty}$,

$$
\left|\alpha^{-1}\right|=\left|P(\lambda)^{-1}\right|<(e+1)^{n} \cdot f^{m}+1 .
$$

Taking the inverse proves our assertion.

We have immediately:

Lemma 3. Let $P$ be an arbitrary integral polynomial of size $s$ and degree $n \geqslant 2$. If $\gamma$ (real or complex) satisfies

$$
P^{\prime}(\gamma)=0, \quad \text { but } P(\gamma) \neq 0,
$$

then

$$
|P(\gamma)|>\left\{n^{n} \cdot(s+1)^{n-1} \cdot s^{n}+1\right\}^{-1}>\left\{n^{n} \cdot(s+1)^{2 n-1}\right\}^{-1} .
$$


Proof. We can apply Lemma 2 with $Q=P^{\prime}, e=s$ and $f=\left|P^{\prime}\right|_{1} \leqslant n \cdot s$.

We know by our intuition (and the Theorem of Rolle), that there is a root $\gamma$ of $P^{\prime}$ between two roots of $P$. If the value of $P$ at $\gamma$ is rather large then, again intuitively, the polynomial needs some space to go from this value to zero. These are the fundamental considerations of the following lower bound for $\operatorname{rsep}(P)$.

THEOREM 2. Let $P$ be an arbitrary integral polynomial (perhaps having multiple zeros) of size $s$ and degree $n$. Then

$$
\operatorname{rsep}(P)>2 \cdot\left\{n^{n+1} \cdot(s+1)^{2 n}\right\}^{-1} .
$$

Proof. Let $P(\alpha)=P(\beta)=0$ such that $\operatorname{rsep}(P)=|\alpha-\beta|$. We distinguish three cases:

(a) $-1 \leqslant \alpha<\beta \leqslant 1$. With $P^{\prime}(x)=\sum_{i=1}^{n} i a_{i} \cdot x^{i-1}$ and $|\mu| \leqslant 1$ we get

$$
\left|P^{\prime}(\mu)\right| \leqslant \sum_{i=1}^{n}\left|i \cdot a_{i} \cdot \mu^{i-1}\right| \leqslant \sum_{i=1}^{n}\left|i \cdot a_{i}\right| \leqslant n \cdot s .
$$

Applying the Theorem of Rolle, we have a $\gamma$ with

$$
\alpha<\gamma<\beta \quad \text { and } P^{\prime}(\gamma)=0
$$

The maximum slope of $P$ in the interval $(\alpha, \beta) \subseteq[-1,1]$ is by (10) less

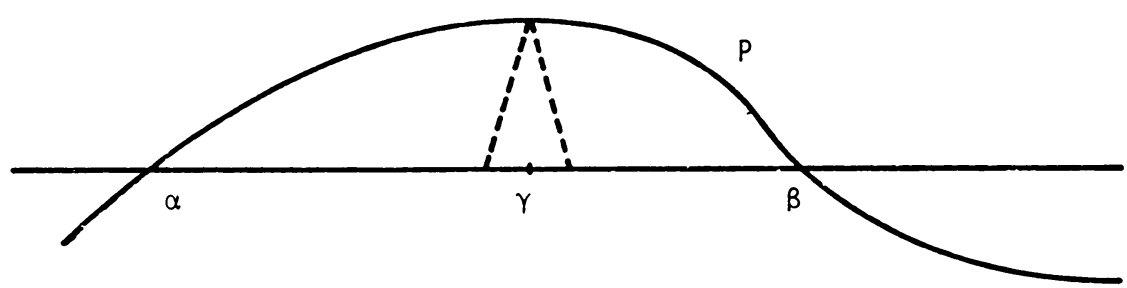

Figure 1

Minimum real root separation

than or equal to $n \cdot s$. So (see Figure 1) applying Lemma 3 yields

$$
|\beta-\gamma| \geqslant|P(\gamma)| \cdot(n \cdot s)^{-1}>\left\{n^{n+1} \cdot(s+1)^{2 n}\right\}^{-1},
$$

and a similar inequality, replacing $\beta$ by $\alpha$, gives the result.

(b) $|\alpha|>|\beta| \geqslant 1$. Take $x^{n} \cdot P(1 / x)=\sum_{i=0}^{n} a_{i} \cdot x^{n-i}$, observe $|\alpha-\beta|>$ $|(\alpha-\beta) / \alpha \beta|=\left|\alpha^{-1}-\beta^{-1}\right|$ and apply (a).

(c) $|\alpha|<1,|\beta|>1$. In replacing, if necessary, $P(x)$ by $P(-x)$ we can write $-1<\alpha<1<\beta$. From the definition of $\operatorname{rsep}(P)=|\alpha-\beta|$ we get $P(1) \neq 0$.

Moreover, $P(1)$ is an integer, so that $|P(1)| \geqslant 1$ holds. Together with (10) we have

$$
|\beta-\alpha|>|\alpha-1| \geqslant|P(1)| /(n \cdot s) \geqslant(n \cdot s)^{-1}>2 \cdot\left\{n^{n+1} \cdot(s+1)^{2 n}\right\}^{-1} .
$$

The distinction between these three cases sharpens the bound with a factor $s^{n}$. It is possible to generalize the result to complex zeros to obtain a lower bound for $\operatorname{sep}(P)$, but we first derive a much better estimation as a basis for a bound of $\operatorname{sep}(P)$. 
THEOREM 3. Let $P$ be an arbitrary integral polynomial (perhaps having multiple zeros) of size $s$ and degree $n$. Then

$$
\operatorname{rsep}(P)>2 \cdot \sqrt{2} \cdot\left\{n^{n / 2+1} \cdot(s+1)^{n}\right\}^{-1} .
$$

Proof. Let $P(\alpha)=P(\beta)=0$ for real $\alpha, \beta$ with $\operatorname{rsep}(P)=|\alpha-\beta|$. As in the proof of Theorem 2 we distinguish three cases:

(a) $-1 \leqslant \alpha<\beta \leqslant 1$. By the Theorem of Rolle we get again a real $\gamma$ with $-1 \leqslant \alpha<\gamma<\beta \leqslant 1$ and $P^{\prime}(\gamma)=0$. We expand $P$ in a Taylor series:

$$
0=P(\beta)=P(\gamma)+\frac{h^{2}}{2} \cdot P^{\prime \prime}(\omega),
$$

where $\gamma<\omega<\beta$ and $h=|\gamma-\beta|$. Therefore

$$
2 \cdot|P(\gamma)|=h^{2} \cdot\left|P^{\prime \prime}(\omega)\right|>2 \cdot\left\{n^{n} \cdot(s+1)^{n-1} \cdot s^{n}+1\right\}^{-1}
$$

by Lemma 3 . On the other hand, $|\omega|<1$, so

$$
\left|P^{\prime \prime}(\omega)\right| \leqslant\left|\sum_{i=2}^{n} i \cdot(i-1) \cdot a_{i} \cdot \omega^{i-2}\right|<n^{2} \cdot s
$$

Combining (13) and (14) yields

$$
h^{2}>2 \cdot\left\{n^{n+2} \cdot(s+1)^{2 n}\right\}^{-1},
$$

and replacing $\beta$ by $\alpha$ in (12) gives the stated result.

(b) $|\alpha|>|\beta| \geqslant 1$. See proof of Theorem 2 .

(c) $|\alpha|<1,|\beta|>1$. We can adjust this case as in the proof of Theorem 2 (with some changes).

4. Lower Bounds for $\operatorname{sep}(P)$. As we remarked in the last number, in the real case it is very easy to find a root of $P^{\prime}$ in the vicinity of two roots $\alpha, \beta$ of $P$ (in fact between them). In the complex plane a "between" does not exist, so our hope is to find a root of $P^{\prime}$ in the circle with diameter $(\alpha, \beta)$. However, we cannot prove this in general, but we can state the following lemma using a theorem due to GraceHeawood in its original version:

LEMMA 4. Let $P$ be an arbitrary complex polynomial of degree $n$. Let $P(\alpha)=$ $P(\beta)=0$ for $\alpha \neq \beta$, where the multiplicities of $\alpha$ and $\beta$ may be greater than one.

Then at least one zero $\gamma$ of the derivative $P^{\prime}$ of $P$ satisfies $P(\gamma) \neq 0$ and

$$
|\gamma-\alpha|<2 n \cdot \epsilon \text { and }|\gamma-\beta|<2 n \cdot \epsilon \text { with } \epsilon=|\beta-\alpha|
$$

Proof. We know from [Mn49, Theorem 25.2] the existence of at least one zero of $P^{\prime}$ in the circle $C$ of

$$
\text { radius } \epsilon \cdot \csc \frac{\pi}{2 n-2} \text { with center } \frac{\alpha+\beta}{2}, \quad \pi=3.14 \ldots
$$


We are finished when we have proved

$$
\frac{1}{2}+\csc \frac{\pi}{2 n-2}<2 n
$$

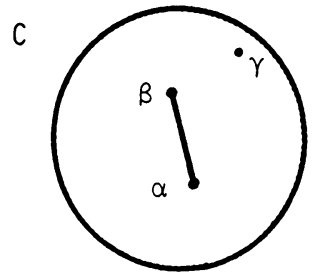

FIGURE 2

Minimum root separation in the complex plane

By Lucas' Theorem (cf. [Mn49, Theorem 6, 1]) we can assume $n \geqslant 3$, hence

$$
\sin \frac{\pi}{2 n-2}>\frac{\pi}{2 n-2} \cdot\left\{1-\frac{\pi^{2}}{(2 n-2)^{2}}\right\}>\frac{1}{3} \cdot \frac{\pi}{2 n-2}=\frac{\pi}{6 n-6} .
$$

Therefore

$$
\frac{1}{2}+\csc \frac{\pi}{2 n-2}<\frac{1}{2}+\frac{6 n-6}{\pi}<\frac{6}{\pi} \cdot n<2 n
$$

so (15) and our lemma is proved.

Lemma 4 shows that the needed zero $\gamma$ of $P^{\prime}$ cannot lie arbitrarily far away.

Now we can use the same idea as in the proof of Theorem 3, expanding $P$ in a complex Taylor series at a root of $\mathrm{P}$ and computing $P(\gamma)$. Because the square root is to be taken we obtain a very good lower bound.

THEOREM 4. Let $P$ be an arbitrary integral polynomial (perhaps having multiple zeros) of size s and degree $n$. Then

$$
\operatorname{sep}(P)>\left\{2 \cdot n^{n / 2+2} \cdot(s+1)^{n}\right\}^{-1} .
$$

Proof. Let $\alpha$ and $\beta$ be zeros of $P$ such that $|\alpha-\beta|=\operatorname{sep}(P)$. If $|\alpha|>1$ and $|\beta|>1$, then $\alpha^{-1}$ and $\beta^{-1}$ are roots of $\bar{P}(x)=x^{n} \cdot P(1 / x)$ and $\left|\alpha^{-1}-\beta^{-1}\right|=$ $|\alpha-\beta| /|\alpha \beta|<|\alpha-\beta|$, so we may assume that either $|\alpha| \leqslant 1$ or $|\beta| \leqslant 1$. Suppose $|\beta| \leqslant 1$, and let $\gamma$ be a root of $P^{\prime}$ for which $|\beta-\gamma|$ is minimal. Then

$$
0=P(\beta)=P(\gamma)+\sum_{i=2}^{n} \frac{h^{i}}{i !} \cdot P^{(i)}(\gamma)
$$

where $h=\beta-\gamma$ and $P^{(i)}$ denotes the $i$ th derivative of $P$. Also,

$$
\begin{aligned}
\left|P^{(k)}(\gamma)\right| & \leqslant \sum_{i=k}^{n}\left|n \cdot(n-1) \cdots \cdot(n-k+1) \cdot a_{i} \cdot \gamma^{n-k}\right| \\
& \leqslant n^{k} \cdot s \cdot \max (1,|\gamma|)^{n} .
\end{aligned}
$$


We may assume that $|\alpha-\beta|<1 / 4 n^{2}$ since otherwise $\operatorname{sep}(P)=|\alpha-\beta| \geqslant 1 / 4 n^{2}>$ $\left\{2 \cdot n^{n / 2+2} \cdot(s+1)^{n}\right\}^{-1}$, proving the theorem. By Lemma $4,|h|=|\gamma-\beta|<$ $2 \cdot n \cdot|\alpha-\beta|<1 / 2 n$. Therefore $|\gamma| \leqslant|\beta|+|\gamma-\beta| \leqslant 1+1 / 2 n$ and $|\gamma|^{n}<$ $(1+1 / 2 n)^{n}<e^{1 / 2}$. Hence by (17) we have

$$
\left|P^{(k)}(\gamma)\right|<e^{1 / 2} \cdot n^{k} \cdot s .
$$

By (16), (17) and (18),

$$
\begin{aligned}
|P(\gamma)| & \leqslant e^{1 / 2} \cdot\left|h^{2}\right| \cdot n^{2} \cdot s \cdot \sum_{i=2}^{n}\left|\frac{(n \cdot h)^{i-2}}{i !}\right|<e^{1 / 2} \cdot\left|h^{2}\right| \cdot n^{2} \cdot s \cdot \sum_{i=2}^{n} \frac{(1 / 2)^{i-2}}{i !} \\
& \leqslant e^{1 / 2} \cdot\left|h^{2}\right| \cdot n^{2} \cdot s \cdot 4 \cdot\left(e^{1 / 2}-1.5\right)<\left|h^{2}\right| \cdot n^{2} \cdot s .
\end{aligned}
$$

By Lemma 3,

$$
\left\{n^{n} \cdot(s+1)^{2 n-1}\right\}^{-1}<|P(\gamma)|
$$

By (19) and (20), $\left|h^{2}\right|>\left\{n^{n+2} \cdot(s+1)^{2 n}\right\}^{-1}$. Hence $|\beta-\alpha|>|h| \cdot 1 / 2 n>1 / 2 n$. $\left\{n^{n / 2+1} \cdot(s+1)^{n}\right\}^{-1}=\left\{2 \cdot n^{n / 2+2} \cdot(s+1)^{n}\right\}^{-1}$, completing the proof.

Some of the estimates in the proof can be sharpened in obvious ways, but this improves Theorem 4 only slightly and would complicate it unnecessarily.

One interesting application of the last theorem is that the imaginary part of a root of an arbitrary integral polynomial is equal to zero or not less than $\left\{4 \cdot n^{n / 2+2}\right.$. $\left.(s+1)^{n}\right\}^{-1}$.

Another sharpening of the estimate in Theorem 4 can be obtained in making further assumptions, e.g., that one can find a nonreal root $\gamma$ of $P^{\prime}$ (see [Gu67]).

5. Best Bounds and Further Research. Suppose $P$ to be an arbitrary integral polynomial of degree $n$ and size $s$. Then for lower bounds of $\operatorname{sep}(P)$ we have just proved

$$
\log \operatorname{sep}(P)^{-1} \leqslant \frac{n+4}{2} \cdot \log n+n \cdot \log (s+1)+c
$$

where $P$ may have multiple zeros and $c$ is a real constant (the basis for the logarithm is 2). Mignotte observed [Mi76], that a corollary of a deep theorem of Schmidt/ Wirsing (cf. [Sm72, Theorem 7I]) can be used to prove

$$
\lim _{s \rightarrow \infty} \frac{\log \operatorname{sep}(P)^{-1}}{\log s} \geqslant\left[\frac{n+1}{2}\right]
$$

for fixed degree $n$ and assuming $P$ to have no multiple roots. This leads to:

Problem 1. Find a better lower bound for $\operatorname{sep}(P)$ or $\operatorname{rsep}(P)$.

In their paper [CL76] Collins and Loos described a new algorithm for isolating the real zeros of an integral polynomial. Its computing time is dominated by

$$
O\left\{n^{10}+n^{7} \cdot \log (n \cdot s)^{3}\right\}
$$


where $O$ denotes Landau's Symbol: $f=O(g) \Longleftrightarrow$ there exists a positive real constant $c$ with $f \leqslant c \cdot g$. Obviously, $\left|\bar{P}^{(i)}\right|_{1} \leqslant 2^{n} \cdot s$ where $\bar{P}^{(i)}$ denotes the primitive part of $P^{(i)}$, so that with Theorem 4 for the $i$ th derivative of $P$

$$
\log \operatorname{sep}\left(P^{(i)}\right)^{-1}=O\left\{n^{2}+n \cdot \log (s+1)\right\}
$$

holds. The summand $n^{10}$ in (21) occurs only because of our inability to omit the summand $n^{2}$ in (22). So we state:

Problem 2. Prove that

$$
\log \operatorname{sep}\left(P^{(i)}\right)^{-1}=O\{n \cdot \log (n \cdot s)\}
$$

holds for every (squarefree) integral polynomial $P$.

The problem is solved if $P$ has only real simple zeros (cf. [Ob63, Satz 5.3]):

LEMma (RIESZ). Let $P$ be an integral polynomial with only real simple zeros.

Then

$$
\operatorname{sep}\left(P^{\prime}\right)>\operatorname{sep}(P)
$$

However, this does not remain true if $P$ has complex zeros (take $P(x)=x^{3}-$ $2 x^{2}+x-2=\left(x^{2}+1\right) \cdot(x-2) ;$ then $\left.\operatorname{sep}(P)=2>2 / 3=\operatorname{sep}\left(P^{\prime}\right)\right)$. Nevertheless, we hope to find something like $\operatorname{sep}\left(P^{\prime}\right)>1 / n \cdot \operatorname{sep}(P)$.

Acknowledgement. I would like to thank the referee for several helpful remarks.

Institut für Angewandte Mathematik

Universität Karlsruhe

Postfach 6380

D-7500 Karlsruhe, West Germany

[Ca47] A. CAUCHY, "Analyse algébrique," in Oeuvres Complètes, II série, tome III, p. 398, formula (48), Paris, 1847.

[CH74] G. E. COLLINS \& E. HOROWITZ, "The minimum root separation of a polynomial," Math. Comp., v. 28, 1974, pp. 589-597.

[CL76] G. E. COLLINS \& R. G. K. LOOS, "Polynomial real root isolation by differentiation,"

Proc. 1976 ACM Symposium on Symbolic and Algebraic Computation, August 1976, pp. $15-25$.

[Ge59] A. O. GELFOND, Transcendental and Algebraic Numbers, Dover, New York, 1959.

[Gu61] R. GUंTING, "Approximation of algebraic numbers by algebraic numbers,"

Michigan Math. J., v. 8, 1961, pp. 149-159.

[Gu67] R. GUंTING, "Polynomials with multiple zeros," Mathematika, v. 14, 1967, pp. $181-196$.

[Kn69] D. E. KNUTH, The Art of Computer Programming, Vol. I (Fundamental Algorithms), Addison-Wesley, Reading, Mass., 1969.

[ $\mathrm{La05}$ ] E. LANDAU, "Sur quelques théorèmes de M. Petrovic̀ relatifs aux zéros des fonctions analytiques," Bull. Soc. Math. France, v. 33, 1905, pp. 251-261.

[Lo73] R. G. K. LOOS, A Constructive Approach to Algebraic Numbers, Stanford University, 27 pages, April 1973.

[Ma32] K. MAHLER, "Zur Approximation der Exponentialfunktion und des Logarithmus, I," J. Reine Angew. Math., v. 166, 1932, pp. 118-136.

[Ma60] K. MAHLER, “An application of Jensen's formula to polynomials," Mathematika, v. 7, 1960, pp. 98-100.

[Ma64] K. MAHLER, "An inequality for the discriminant of a polynomial," Michigan Math. J., v. 11, 1964, pp. 257-262.

[Mi74] M. MIGNOTTE, “An inequality about factors of polynomials," Math. Comp., v. 28, 1974, pp. 1153-1157. 
[Mi76] M. MIGNOTTE, "Some problems about polynomials," Proc. 1976 ACM Symposium on Symbolic and Algebraic Computation, August 1976, pp. 227-228.

[Mn49] M. MARDEN, The Geometry of the Zeros of a Polynomial in a Complex Variable, Math. Surveys, no. 3, Amer. Math. Soc., Providence, R. I., 1949.

[Ob63] N. OBRESCHKOFF, Verteilung und Berechnung der Nullstellen Reeller Polynome, VEB Deutscher Verlag, Berlin, 1963,

[Ru76] S. M. RUMP, "Isolierung der reellen Nullstellen algebraischer Polynome," Bericht der Arbeitsgruppe Computer Algebra, Universität Kaiserslautern, v. 10, 1976, 121 pp.

[Sm72] W. M. SCHMIDT, Approximation to Algebraic Numbers, Monographie 19 de l'Enseignement Mathématique, Genève, 1972. 1957.

[Sn57] T. SCHNEIDER, Einführung in die Transzendenten Zahlen, Springer-Verlag, Berlin,

[vW66] B. L. van der WAERDEN, Algebra, Springer-Verlag, New York, 1966. 